\title{
DÜBLIN
}

Technological University Dublin ARROW@TU Dublin

\section{An Introduction To Emotional Intelligence}

Aiden Carthy

Technological University Dublin, aiden.carthy@tudublin.ie

Ailish Jameson

Technological University Dublin, ailish.jameson@tudublin.ie

Follow this and additional works at: https://arrow.tudublin.ie/ncrpbk

Part of the Educational Psychology Commons, and the Psychology Commons

\section{Recommended Citation}

Carthy, A. (principal author) and Jameson, A. (2016) An Introduction To Emotional Intelligence. Chapter 1 of book entitled The Emotionally Intelligent College: Transforming Third level Education to help Students and Educators Reach their Maximum Potential, Cambridge Scholar, 2016.

This Book Chapter is brought to you for free and open access by the Research Centre for Psychology, Education and Emotional Intelligence at ARROW@TU Dublin. It has been accepted for inclusion in Book/Book Chapter by an authorized administrator of ARROW@TU Dublin. For more information, please contact arrow.admin@tudublin.ie, aisling.coyne@tudublin.ie, gerard.connolly@tudublin.ie.

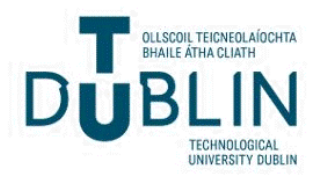




\section{The Emotionally \\ Intelligent College}





\section{The Emotionally Intelligent College:}

Transforming Third Level Education to Help Students and Educators Reach their Maximum Potential

By

Aiden Carthy with Ailish Jameson

Cambridge

Scholars

Publishing

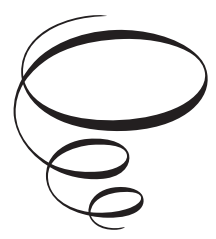


The Emotionally Intelligent College:

Transforming Third Level Education to Help Students and Educators

Reach their Maximum Potential

By Aiden Carthy with Ailish Jameson

This book first published 2016

Cambridge Scholars Publishing

Lady Stephenson Library, Newcastle upon Tyne, NE6 2PA, UK

British Library Cataloguing in Publication Data

A catalogue record for this book is available from the British Library

Copyright (C) 2016 by Aiden Carthy with Ailish Jameson

All rights for this book reserved. No part of this book may be reproduced, stored in a retrieval system, or transmitted, in any form or by any means, electronic, mechanical, photocopying, recording or otherwise, without the prior permission of the copyright owner.

ISBN (10): 1-4438-8689-0

ISBN (13): 978-1-4438-8689-5 


\section{TABLE OF CONTENTS}

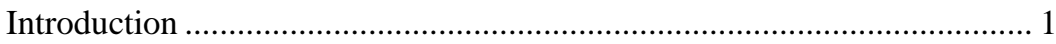

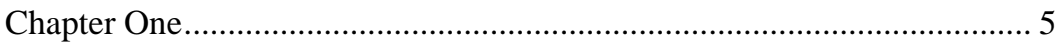

An Introduction to Emotional Intelligence

Chapter Two 34

Emotional Intelligence, the Graduate and the Workplace

By Ailish Jameson

Chapter Three 75

Emotional Intelligence and Academic Attainment

Chapter Four. 102

Developing Emotional Intelligence in Educational Settings

Chapter Five 132 Improving Students' Interpersonal Emotional Intelligence

Chapter Six 156 Improving Students’ Intrapersonal Emotional Intelligence

Chapter Seven. 170 Future Directions 



\section{INTRODUCTION}

If you are planning for a year, sow rice,

if you are planning for a decade, plant trees,

if you are planning for a lifetime, educate people!

(Chinese proverb)

Few would deny the central role that formal education plays in shaping our lives, both at a personal and a societal level. Traditionally, education systems, particularly in Western Europe have focused on helping students to enter the workforce and therefore the emphasis has been on the development of cognitive capacities. At an individual level, encouraging students to reach their maximum cognitive potential increases their career prospects and at a societal level, increased educational standards can lead, in turn, to increased economic power and prosperity. However, in recent decades there has been increasing interest in the role of formal education in empowering personal growth and social and moral development. This has stimulated much debate and encouraged researchers to examine the efficacy and challenges that pertain to encouraging students' social and emotional skills development. A robust body of knowledge has now been amassed that supports the assertion that helping students develop such skills can lead to many advantages including enhanced levels of wellbeing, increased levels of educational attainment and decreased levels of school drop-out. Nevertheless, until recently, much of the research that has been conducted in this area has pertained to younger, primary and second level students. Although there have been extremely valuable and enlightening research projects that have been conducted in third level learning environments, for the most part, such projects have tended to focus on specific subject areas or student populations. There is need for a single volume that synopsises research in this area. Therefore, this text aims to provide a comprehensive synthesis of available theory and research data to enable both students and educators to use emotional intelligence (EI) to help them reach their maximum potential. 


\section{What are the principal aims of this text?}

(1) To provide a brief history of the development of EI and its use in educational settings and to justify the need to incorporate the development of social and emotional skills into third level curricula.

(2) To explore some of the various ways in which EI can be incorporated into academic curricula and national/international qualification frameworks.

(3) To outline some of the barriers that may pertain to encouraging emotional and social skills development in third level settings and to propose solutions accordingly.

(4) To provide a range of exercises and activities that can be employed by students and educators to help them realise their full academic potential and to better prepare them for the workplace.

In essence, this text aims to equip students and educators with key knowledge and skills that can be employed to help them reach their maximum potential. Specifically, it is hoped that the knowledge and advice outlined in this book will encourage student engagement, reduce levels of student drop-out and lead to increased levels of well-being for students. In other words, it is hoped that this book will help to create emotionally intelligent colleges!

\section{Who is this book aimed at?}

This book is aimed at:

- Third level (university/college) lecturers/professors who have an interest in helping students reach their maximum potential.

- Third level students who wish to improve their social and emotional competencies i.e. to become more emotionally aware, better at managing stress and better able to have healthy and fulfilling social relationships. In turn, developing such skills should help you to become a more productive and focused student.

- Theorists who have an interest in the fields of education and/or emotional intelligence. 


\section{Structure of the book}

This book consists of seven chapters as follows:

Chapter one - This chapter introduces the reader to key concepts in the field of emotional intelligence. A brief history of emotional intelligence is provided and following on from this, current issues and debates in the field of EI are comprehensively examined.

Chapter two - This chapter explores the perspectives of employers and how best to prepare graduates for the workplace. A number of key questions are addressed in this chapter, such as, what are the social and emotional skills that employers most value and how might such skills be developed so that graduates maximise their chances of employment?

Chapter three - This chapter explores the costs and benefits of encouraging the development of students' social and emotional competencies. For example, how have researchers attempted to develop such skills in the past, what are the most successful means of helping students to develop these skills and what barriers may pertain to doing so?

Chapter four - This chapter presents potential solutions to the most prevalent barriers that have been found with respect to EI skills development in third level settings. Specifically, a set of recommendations is provided for educators who may wish to consider the provision of EI coaching or of infusing emotional and social skills development into the curricula they teach.

Chapters five and six - These chapters present simple exercises and activities for use in classroom settings that are designed to help students develop a range of key aspects of emotional intelligence. Chapter five focuses specifically on the development of interpersonal aspects of EI and stress management. Chapter six focuses on intrapersonal skills.

Chapter seven - This chapter outlines a number of areas where there is need for future research. Five specific areas where gaps in current knowledge have been identified will be outlined and in each instance, a recommendation for future research is made. 
As stated above, the paramount aim of this book is to help students and educators reach their maximum potential and in doing so to help encourage the development of emotionally intelligent colleges! 


\section{CHAPTER ONE}

\section{AN INTRODUCTION TO EMOTIONAL INTELLIGENCE}

\section{Chapter outline}

Although a comparatively new subject of study, psychology is firmly established as an academic discipline and widely taught in universities and indeed often introduced to second level students worldwide. Yet students are often surprised to discover that there is considerable ongoing debate as to how psychology should be defined and what exactly the nature and content of study within this discipline should be. In particular, there is much debate as to whether psychology should be considered a true science akin for example to chemistry or biology, or should be considered a social science and aligned with subjects such as sociology. Researchers have taken varying positions with respect to this debate depending on a range of factors including, but not limited to, the specific area of study or subdiscipline within the field of psychology that is being investigated, as well as other less tangible social and cultural factors. An in-depth discussion of this debate is beyond the remit of this text. However, what should be clear is that answering the question 'what is psychology?' is not quite as straightforward as one might imagine and this question, as we shall see, is particularly relevant to the study of intelligence. Therefore, chapter one begins with a brief discussion of this issue, following from which, a brief history of emotional intelligence will be provided. The second section of this chapter outlines and discusses some of the principal issues and debates pertaining to this field and finally, an outline of the principal tests of emotional intelligence that are predominantly currently employed in research and practise will be provided. Overall, the primary purpose of this chapter is to provide a solid foundation, particularly for those who may not be familiar with the field of emotional intelligence. Once this has been achieved, readers will be equipped with all of the knowledge and information they require to enable them to specifically explore how 
developing emotional and social skills can benefit students and educators in diverse learning environments.

\section{Psychology/intelligence, a background:}

When the history of psychology is being outlined to students, one of the first things they are ordinarily taught is that psychology developed as a consequence of the marriage of philosophy and physiology. The questions that philosophers had posed pertaining to the nature of the human condition combined with the biological techniques developed by physiologists, led to the development of modern psychology as a distinct, scientific discipline. The separate influences of both philosophy and physiology on the evolution of psychology as a legitimate academic discipline are quite apparent. In particular, throughout the early development of psychology, although admittedly somewhat of a generalisation, many of the first psychologists and indeed many of the first schools of psychological thought, aligned themselves predominantly with either one subject area or the other. Obvious examples are the school of psychoanalysis, which adopted an experiential, subjective approach, and that of behaviourism which adopted an arguably more objective approach. In more recent years although the boundary between the philosophical and physiological aspects of psychological research have become considerably blurred, this distinction is still reflected in the official definition of psychology as the scientific study of experience and behaviour. However, as previously stated, there is in fact considerable ongoing argument as to the extent to which psychology can be considered a science and this debate is particularly relevant to the study of intelligence.

Fundamental issues and questions arise as a consequence of the very different influences that philosophy and physiology have had on the development of psychology. For example, at the simplest level of enquiry, human behaviour is visible and relatively easily recorded and quantified, whereas human experience is subjective and less easily accessible to scientific measurement (psychologists can see with the naked eye how you behave but cannot easily determine what it is that you 'feel'). This means that particularly in the early decades of research in the field of psychology, experience was often considered less amenable to scientific analysis than behaviour. This issue is particularly relevant to the topic of emotional intelligence because whilst intelligence, one's ability to reason, was and still is, measured via observation or standardised tests, emotion is not so easily quantified. Therefore, it is no surprise that traditionally intelligence 
(IQ) has largely been understood in terms of cognitive abilities such as memory skills, problem solving and concrete and abstract reasoning, all of which are areas that are amenable to measurement. The measurement and comparison of such psychological abilities is referred to as 'psychometrics' and whilst areas such as personality are also assessed psychometrically, typically this area focuses on quantifying cognitive reasoning skills. There is undoubted value in doing so. Once a given individual, or indeed population's, IQ is known, comparisons can be made which may enable help and support to be provided for those who may require it. Alternatively, psychometric testing may identify those who possess superior cognitive abilities. From an individual perspective, psychometric testing can help increase self-knowledge by enabling one to identify personal cognitive strengths that may be capitalised upon and weaknesses that may be addressed. The most obvious advantage of doing so is with respect to career choice. Psychometric testing may help one to choose a career path aligned with key strengths, which is naturally of value not only to the individual but also to employers, educators and arguably society as a whole. A robust body of knowledge has been amassed that clearly demonstrates the validity and stability of psychometric measurement and has additionally affirmed a positive relationship between high IQ and academic attainment (e.g. Gottfredson 1998; Laidra, Pullmann and Allik 2007) i.e. that has shown that students with higher levels of IQ, tend also to have better grades. There is clearly much value in measuring student's IQ and in using this knowledge to help support students in their learning. However in recent years many researchers and academics have criticised a perceived over-reliance on the measurement of IQ in academic settings.

\section{Emotional intelligence - a brief history}

Although throughout the early history of intelligence research the focus was on the measurement of cognitive skills, this approach has also attracted some criticism. One such long-standing criticism of the traditional psychometric approach is that many theorists consider this approach limiting and argue that, although IQ scores have a certain stability and validity, they ignore many other important aspects of human functioning (e.g. Wechsler 1943; Sternberg 1985; Bar-On 2007, cited from Bar-On, Maree and Elias 2007). Bar-On 2007 has stated that

'It has been argued for nearly a century that something else is missing in the human performance formula that makes it difficult for us to understand why some people in life do well while others do not, irrespective of how cognitively intelligent they are.' ( p. 1). 
In this regard, as early as 1943, building on the work of Thorndike, Wechsler began to speak of non-cognitive factors of intelligence:

I have tried to show that in addition to intellective there are also definite non-intellective factors that determine intelligent behaviour. If the foregoing observations are correct, it follows that we cannot expect to measure total intelligence until our tests also include some measures of the non-intellective factors.' (p. 103).

He later defined intelligence as 'The aggregate or global capacity of the individual to act purposefully, to think rationally, and to deal effectively with his environment' (1958, 7). Robert Sternberg (1985) more recently proposed a 'Triarchic' theory of intelligence, in which he argued that intelligence is comprised of three separate facets; analytic, practical and creative. Sternberg's work is significant, as it was one of the first major theories of intelligence to include both cognitive and non-cognitive variables. It is also, therefore, one of the most comprehensive theories of intelligence. Sternberg's work helped to widen the scope of intelligence, as he argued that IQ tests only measure specific aspects of intelligence and do not assess social and emotional factors that impact everyday functioning. Over time, as theorists such as Weschler and Sternberg began to question the efficacy of the psychometric approach and as a more substantial body of research was undertaken in this respect, researchers began to realise that intelligence was far from easy to quantify, or even to define. For example, Sternberg and Detterman, in 1987 asked 24 leading experts in the field to provide a definition of the word 'intelligence' and received twenty four different definitions! Accordingly, Bar-On (1997) claimed that it is obviously easier to measure intelligence than it is to define it, since researchers clearly encounter difficulties defining intelligence, yet still prodigiously use intelligence testing in varied settings. In addition, several theorists have argued that rather than being a unitary construct, intelligence is in fact comprised of a number of separate but related constructs and that we should speak not of intelligence in the singular, but of multiple intelligences. For example, Howard Gardner (1983) suggested that intelligence is multi-dimensional and comprises both cognitive and emotional aspects. In his seminal text 'Frames of Mind', he argues in favour of multiple intelligences and proposed that there are eight distinct forms of human intelligence as follows:

- Linguistic intelligence - This area pertains to verbal abilities, both spoken and written, including oration, debating, reading, writing and memory for names and dates. 
- Logico-mathematical intelligence - This area pertains to numerical and reasoning abilities, including pattern recognition, scientific investigation and numeracy.

- Spatial intelligence - This area pertains to abilities to visualise and mentally manipulate objects and includes, hand-eye coordination, visual memory and sense of direction.

- Bodily-kinaesthetic intelligence - This area pertains to physical abilities, including sport, dance and building or making objects.

- Musical intelligence - This area pertains to auditory and musical abilities, including rhythm, composing and musical performance.

- Interpersonal intelligence - This area pertains to the ability to interact with other people and includes empathy, leadership skills and communicative abilities.

- Intrapersonal intelligence - This area pertains to self-awareness and includes, introspection, emotional self-awareness and self-reflection.

- Naturalist intelligence - This area pertains to awareness of natural phenomena, including weather patterns, the ability to nurture animals and crops and classifying objects.

Following from the work of theorists such as Weschler and Gardner, Daniel Goleman in 1996 published the first edition of his best-selling text 'Emotional Intelligence: Why it can matter more than IQ'. Goleman's work was transformational as it helped to create public awareness of the concept of emotional intelligence. Goleman also examined various applications for emotional intelligence theory and practise, including the use of EI in educational settings. In so doing, he demonstrated the value of focusing on broadening educational curricula to include emphasis on the development of students' social and emotional skills development. In turn, Gardner developed a method of individualistic schooling, arguing that if individuals are encouraged to attain vocational goals appropriate to their particular intelligence profiles and are mindful of their individual strengths and weaknesses, they will become more competent and more inclined to serve society in a constructive manner. In 2006, he later claimed that his aim is to present ' ... a pluralistic view of mind, recognizing many different and discrete facets of cognition, acknowledging that people have different cognitive strengths and contrasting cognitive styles' (p. 5).

Evidently, theorists are not in agreement as to whether intelligence comprises one, or many facets and tend to be colloquially referred to as 'lumpers' or 'splitters' (Sternberg 1990); hence, Gardener for example, would fall into the latter category. 'Lumpers' define intelligence as a 
general unified capacity and the notion that intelligence is a unitary construct implies that it can be measured and represented by a single value. Intelligence is therefore commonly referred to by such theorists simply as ' $\mathrm{g}$ ' to signify 'general intelligence'. Splitters, on the other hand, hold that intelligence is comprised of many separate abilities, including emotional and social capacities, which operate independently and, therefore, that emotional intelligence can be measured separately from cognitive intelligence. Although a majority of researchers have traditionally held that intelligence is a unitary construct, in recent years as the concept of multiple intelligence has gained in popularity, there are now quite distinct bodies of research pertaining to various different aspects of intelligence, including emotional intelligence.

The notion of multiple intelligences certainly has an intuitive appeal and appears to align with what psychologists would refer to as our "natural schema of correspondence', or real-world understanding. Why is it then that standard, cognitive based IQ tests have become so popular? Gardner (1983) claimed that such popularity may be explained by the ease with which they allow us to categorise individuals. People had always, he claimed, intuitively assessed the intelligence of others in an attempt to categorise and compare them and with the development of intelligence testing, were provided with a simple means of doing so. Weinberg (1989), further argued that the perceived need for I.Q. tests in industrial societies was, and indeed still is, due to a rise in the level of formal education, diverse job types and the rapid growth of cities, forcing the need to make rapid 'selection decisions'. Gardner (1993) has also identified the following three ideologies in western society which he argues, serve to perpetuate the use of IQ tests:

(1) 'Westist' - placing certain western values on a pedestal, for example logico-mathematical skills, merely because they imply high scores on I.Q. tests;

(2) 'Testist' - focusing on human abilities which are readily testable and which may lead to the undervaluing of less defined global human abilities such as interpersonal skills; and

(3) 'Bestist' - the belief that all of the answers to a given problem will lie within a particular theory or dimension.

In addition to the above, the notion of intelligence as a stable construct as espoused by the traditional cognitive model of intelligence has been challenged. For example, the cognitive model of intelligence assumes IQ 
to be relatively stable across the lifespan and as such, holds that IQ levels cannot increase dramatically as a consequence of age or experience (although as a consequence of illness or trauma, cognitive functioning may certainly decline). In contrast, research has demonstrated that emotional and social skills can be taught and can improve as a result of training or experience (e.g. Côté and Miners 2006; Boyatzis and Saatcioglu 2008; Nelis, Quoidback, Mikolajczak and Hansenne 2009; Carthy, McCann, McGilloway and McGuinness 2013). For example, Nelis et al. (2009) provided a total of 10 hours EI coaching to a small sample of psychology students $(\mathrm{n}=19)$, whilst a matched sample $(\mathrm{n}=18)$ received no intervention. Although the sample size for this study was quite small, results revealed that there were statistically significant increases in emotional identification and emotion management for students that received coaching compared to those who did not. Importantly, when follow-up measures were taken six months following the initial training, improvements in EI were still present. The authors conclude that

'results suggest that traits that have shown to be relatively stable over time can be modified through intensive training. However, as these traits are relatively stable, it is possible that people will come back to their 'baseline' after a while if the competencies are not practised'. ( $\mathrm{p} 40)$.

This is a very important distinction between the cognitive and emotional intelligence models and in fact the assumption that EI can be taught and improved forms the very basis for this text!

The debates outlined above suggest that emotional and cognitive intelligence are distinct, yet related, aspects of human intelligence and this supposition is importantly also supported by neurobiological research which affirms the notion of intelligence as a pluralistic rather than a singular construct and suggests that emotional intelligence is indeed separate, yet related to, cognitive functioning. The following section discusses this in more detail.

\section{Emotional Intelligence - Biological Substrates}

Gardner's theory of multiple intelligences argues in favour of emotional intelligence as an evolved mechanism. For example, each form of intelligence, it is claimed, is a unique, universal human skill and is, therefore, assumed to have a distinct biological basis. Although research evidence has not, as yet, uncovered separate neural pathways that are implicated in each distinct aspect of emotional intelligence, there is evidence to suggest the existence of a neural circuit involving the 
amygdala and the pre-frontal, somatosensory and ventromedial cortex that is implicated globally in social/emotional reasoning and that operates separately from brain circuitry implicated in cognitive reasoning (Bar-On et al. 2003; Damasio 1994; Reis et al. 2007). For example, Reis et al. (2007) used neuro-imaging techniques to measure brain activation when participants were either presented with a task related to social reasoning or a matched non-social reasoning task; they found that there were different patterns of activation recorded for both types of task with successful social reasoning related to increased activity in the pre-frontal cortex and the frontal and temporal cortices. Damasio (1994) compared the cognitive and social functioning of patients with lesions either to the ventromedial cortex, the amygdala or the somatosensory cortex and also compared all three groups to a control group. Results revealed that there were no significant differences between the control and any of the three experimental groups with respect to cognitive functioning whereas the social reasoning of members of all three experimental groups was impaired i.e. the lesions that patients in all three experimental groups suffered had not impaired their cognitive capacities, but had impaired their social reasoning, thereby lending support to the hypothesis that social and cognitive functioning are controlled by separate neural circuitry. Domasio also points out that cognitive intelligence, from a neurobiological perspective, provides more of a 'pure case' than EI in the sense that the brain structures that are involved in cognitive reasoning are thought to be relatively localised, whereas emotional and social reasoning is more distributed in neural terms.

Although research suggests that emotional and cognitive reasoning are controlled by separate neural structures, there is also some evidence to suggest that there are many connections between the emotional and cognitive centres of the brain. For example, Goleman (1996) claims that many of the brain areas which are implicated in both concrete and abstract reasoning have evolved from the limbic system, which is the emotional centre of the brain. Throughout our recent evolution, as the neo-cortex began to develop, it did so by branching out from the limbic system and, therefore, there is a vast number of connections between the emotional centres of the brain and all parts of the cortex. This, in essence, gives the emotional centres of the brain considerable influence over our faculties of self-awareness, reasoning and deduction (Beatty 1995), and suggests that there is some overlap between emotional and cognitive reasoning and that our perception of the world is influenced both by cognitive and emotional factors (Barrett, Mesquita, Ochsner and Gross 2007). Davisson, Jackson 
and Kalin (2000) have also found that, even in adulthood, the brain circuitry involved in emotional reasoning shows a reasonable degree of plasticity, which is an important finding as it indicates that emotional learning can occur throughout the lifespan.

Thus, although separate neural pathways have not been uncovered for each of the separate intelligences posited by multiple intelligence theorists such as Gardener, neurobiological evidence would appear to generally support the multiple intelligence perspective, which holds that emotional and cognitive capacities are both separate, yet related, aspects of human intelligence. As there is some degree of overlap between the neural circuitry implicated in emotional and in cognitive reasoning, neurobiological evidence highlights the reciprocal nature of both aspects of intelligence. For example, Humphrey, Curran, Morris, Farrell and Woods (2007), outline the importance of the neurochemical dopamine in bridging the emotional and rational aspects of reasoning at a biochemical level. They assert that emotions impact goal setting behaviours and problem solving as they act as a filter that focuses our attention on the problems that most need to be addressed at any given moment in time. This, they argue,

\begin{abstract}
'closely parallels our understanding of brain physiology and especially the control of dopamine release. Dopamine release first switches our attentional system to a particular stimulus and then facilitates cognitive activation through its release in the frontal brain, before finally facilitating the passage of relevant information throughout the brain and establishing learning.' (p 237-238).
\end{abstract}

It is precisely this interrelationship between the cognitive and emotional systems at a physiological level that highlights the importance of enabling students and educators to develop both rational and emotional capacities. In this regard, Salovey and Mayer (1990) defined emotional intelligence as the ability to recognise and monitor one's emotions and to use this information 'to guide one's thinking and actions'. An awareness of and ability to control emotional responses can therefore act as a guide to thinking and action, just as cognitive problem solving abilities can reciprocally aid the recognition of patterned emotional responses and thereby facilitate emotional awareness. Particularly for the benefit of readers who may not have background knowledge pertaining to neuroanatomy and/or neuropsychology, in order to simplify the above, a graphical representation of the relationship between EI, IQ and multiple intelligence is provided (Figure 1.1). The strong link between cognitive and emotional functioning and the malleable nature of emotional 
reasoning highlights, from an educational perspective, the importance of fostering both cognitive and non-cognitive functioning in the classroom.

\section{Current issues in emotional intelligence research}

Matthews, Zeidner and Roberts (2008) provided a useful summary of the three most important current issues that they perceived to exist at that time with respect to the measurement and use of EI and interestingly Murphy (2013) has more recently provided a summary of the key issues as he sees them and listed exactly the same three areas Matthews et al. originally provided. These three issues are; (1) there is no consensually agreed definition of EI; (2) it is unclear to what extent EI is cognitive in nature and; (3) there is no consensus as to how exactly EI should be measured or how stable a construct it is. Given that there appears to be somewhat of a consensus that these are the three principal issues that pertain to the measurement of EI, each will now be discussed in more detail.

\section{Defining EI}

As the field of emotional intelligence has developed, one of the principal issues facing researchers, was to demonstrate the construct validity of EI by proving that tests of EI were measuring unique aspects of human experience and behaviour that were not already accounted for by preexisting measures. In this regard, researchers have debated the extent to which tests of EI have incremental validity over more traditional tests of IQ and of personality. As highlighted above, both experimental and neurobiological evidence suggests that EI and IQ are distinct, but related constructs. Nevertheless, Emmerling and Goleman (2003) have noted that it can be difficult to separate abilities related to cognitive and emotional intelligence, whilst definitions of emotional intelligence also usually include reference to cognitive ability. Conceivably, although EI should emerge as a separate construct when mapped against IQ, all tests of EI should also correlate to some extent with traditional tests of IQ, as EI is after all, classified as a form of intelligence. Cherniss and Goleman (2011) discuss this issue and assert that there is general consensus amongst EI researchers that EI and IQ do correlate, although there is still some debate as to the extent to which this occurs. 


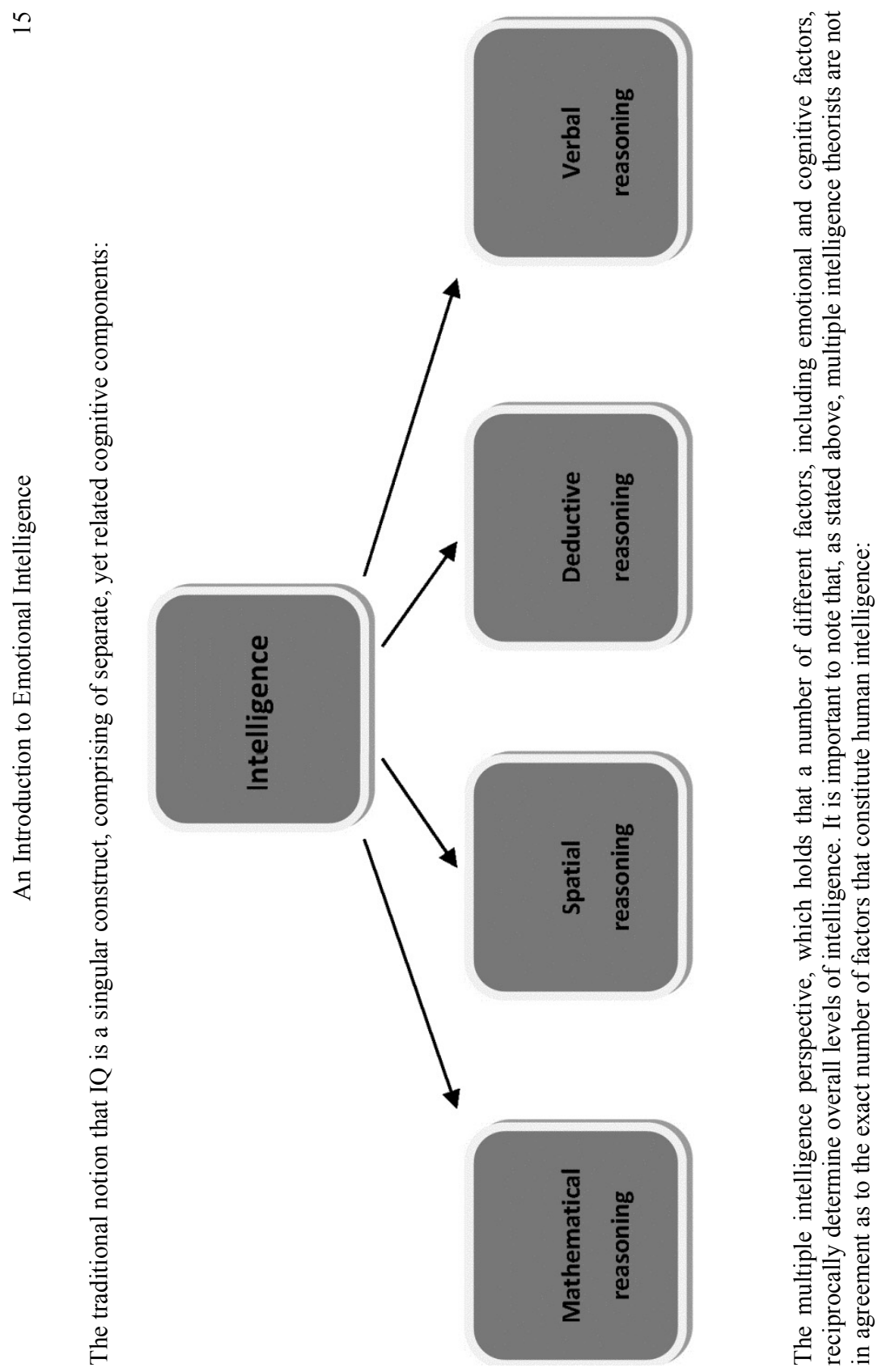




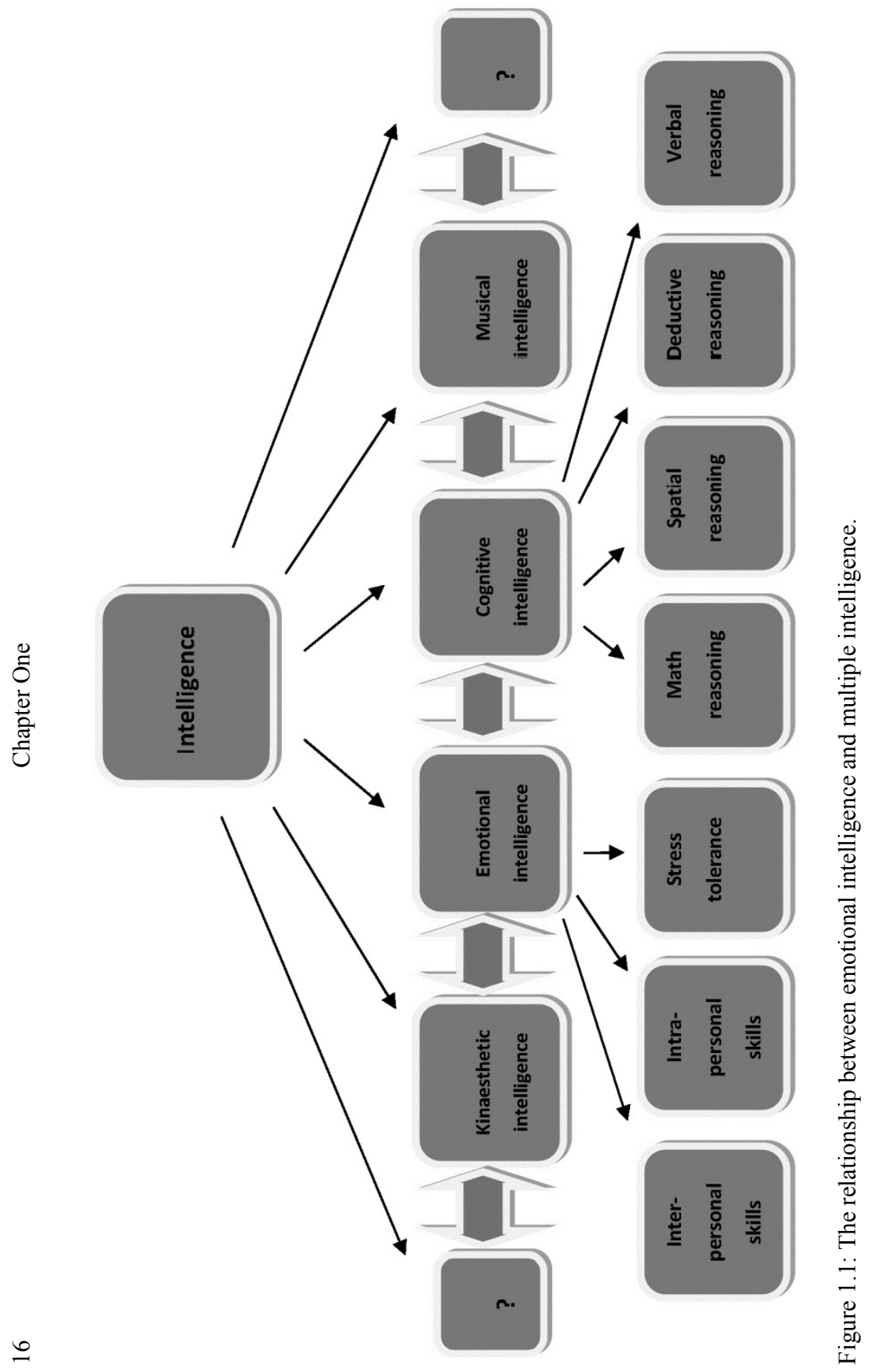


However, the debate as to the extent to which tests of EI should correlate with tests of personality is much more contentious. Although research has found that emotional intelligence emerges as a unique construct when personality characteristics are controlled for (Van Rooy and Viswesvaran 2004; Rosete and Ciarrochi 2005; Joseph and Newman 2010), the extent to which EI and personality overlap, appears to vary according to the test of EI that is employed (Emmerling and Goleman 2003). In order to further address some of the conceptual debates about EI and its measurement, it is first necessary to outline the three different theoretical frameworks within which tests of EI traditionally fall. These include:

- Theories built on the assumption that emotional intelligence is ability-based.

- Theories built on the assumption that emotional intelligence is trait- or competency- ${ }^{1}$ based.

- Mixed models built on the assumption that emotional intelligence is both ability and trait based.

In line with the above, one of the principal debates central to the development of measures of EI, is whether emotional intelligence is ability or trait based. If ability-based, emotional intelligence is assumed to have a strong genetic component and, therefore, to be somewhat resistant to change. If emotional intelligence is assumed to be trait-based, it is considered to be similar to personality, which may arguably be more amenable to social learning and more readily changed through experiential learning. Some theorists have also produced mixed models of EI which adopt an interactionist stance by claiming that EI is partly genetically influenced, but also open to a certain degree of social influence. Essentially, within the field of EI, it is generally acknowledged that tests derived from ability-based theories of EI, which assume EI to be genetically based, will not greatly overlap with tests of personality, but will overlap to some degree with tests of IQ. Conversely, tests developed from trait-based theories of EI, which assume EI to be amenable to social learning, will overlap to a lesser extent with tests of IQ, but will have a greater degree of overlap with personality tests (Emmerling and Goleman 2003; Saklofske, Austin and Minski 2003). Furthermore, whilst researchers have not reached a consensus as to the extent to which EI is in fact trait- or ability- based, it is important to note that the great majority of

\footnotetext{
${ }^{1}$ Both terms are employed interchangeably in literature pertaining to emotional intelligence.
} 
theorists do recognise that, to some extent, both tests of ability and traitbased tests of EI are measuring valid aspects of emotional intelligence, because both genetic and social factors will impact the development and expression of EI.

However, it is important to remember that, although some overlap exists between EI and IQ and between EI and personality, all three constructs have been demonstrated to be relatively orthogonal in nature, as research has shown high discriminant validity for all three (Van Rooy and Viswesvaran 2004) i.e. research has found that all three are separate (albeit related) constructs. For example, recently, Pérez-González and SanchezRuiz (2014) examined the relationship between trait EI and personality (the Big Five, the Big Two and the Big One/General Factor of Personality $\left.(\mathrm{GFP})^{2}\right)$. A sample of university students $(\mathrm{n}=289)$ agreed to have their personality and trait EI measured by the NEO PI-R and TEIQue respectively. The data that was collected was subjected to a thorough analysis involving a range of statistical measures. Therefore, in the interest of simplifying the results from this study for readers who may not have a comprehensive knowledge of statistics, the results are summarised as follows (readers who are interested in acquiring a more thorough knowledge of this research are encouraged to consult the original article). Results revealed that although trait EI and the Big Five were strongly correlated (although the correlation for agreeableness was comparatively low at $\mathrm{r}=.16$ ), trait EI also emerged as a separate aspect of personality not fully accounted for by the Big Five. Trait EI was also found to positively

\footnotetext{
${ }^{2}$ Theorists in the field of personality have established that there are five aspects of human personality that tend to remain particularly stable across the lifespan. For this reason, these personality factors have traditionally been referred to as the Big Five (extraversion, neuroticism, agreeableness, openness to new experiences and conscientiousness). In recent years psychologists have also begun to refer to a Big Two whereby the Big Five are amalgamated into two super personality structures consisting of an Alpha structure (neuroticisn, agreeableness and conscientiousness) and a Beta structure (Extraversion and Openness to new experiences). Still other theorists have referred to a Big One/GFP which is similar to the concept of $\mathrm{g}$ with reference to intelligence. There is less empirical evidence to support the validity of the Big Two and less again to support the Big One, than there is to support the Big Five. In fact, there is some debate as to whether the GFP is in fact a valid construct at all. Nevertheless, there is some evidence to support the idea that there are five generally stable aspects of personality (the Big Five), which are subsumed under two more general aspects (the Big Two) which are in turn subsumed under one global personality construct (the Big One). However much further research is required in this area to ascertain how, if at all, such constructs align and interact.
} 
correlate with the Big Two and the Big One. In essence, this research appears to confirm the hypothesis that trait EI and personality are separate, yet strongly related constructs.

Brackett and Mayer (2003) also compared trait- and ability-based tests of EI and found good discriminant validity between them, which argues that both types of test may tap different aspects of EI. These issues may be resolved in future work, and the relative contribution of genetic and social factors and the extent to which trait and ability measures of EI overlap, may be more fully understood, leading to the development of more predictive and robust measures. For example, if trait-based and abilitybased tests do indeed measure different aspects of EI, future tests may combine some of the features of both. In this regard, Matthews, Zeidner and Roberts (2008), claim that 'There would appear a need to derive more unified, full-blown theories of emotional intelligence, models that would embrace developmental, neurobiological, genetic and behavioural components.' (p. 465). Also Boyatzis (2009) cautions that multiple traits may present as the same ability; for example, one may engage in active listening arising from a developed sense of empathy, or from a feeling of mistrust and hence a desire to seek weaknesses or flaws in the information that is being presented by a speaker. In either case, the ability (active listening) will be identical, whereas the traits (empathy versus mistrust) will not. Therefore, he posits that a multi-level approach to emotional intelligence should be adopted, which accounts both for emotional abilities and emotional traits.

In recent years, some theorists have also begun to refer to emotional selfefficacy (ESE) (Dacre Pool 2013; Kirk, Schutte and Hine 2008) which refers to the level of confidence that individuals may possess with respect to their emotional competencies i.e. how emotionally intelligent one may believe they are. As of yet, there is not a wide body of research that has been conducted that has specifically assessed this aspect of EI, although there is growing interest in this area. However preliminary research does suggest that students' levels of ESE can be improved (Dacre Pool and Qualter 2012). Arguably, ESE may not necessarily be a separate form of emotional intelligence but merely a synonym for emotional self-awareness which is an aspect of EI that is tested for with some standard tests of emotional intelligence, as detailed below. Although further research is required in this area, there is no doubt that increasing both students' emotional competencies and their confidence to employ such competencies is of value and this is discussed further in chapter three. 


\section{The measurement of emotional intelligence}

As indicated above, those who propose that EI is an ability are seeking a certain degree of convergent validity with intelligence tests (i.e. on the assumption that if someone possesses high levels of EI, they should also possess high levels of IQ) and will tend to measure it through the use of performance-based tests. However, those who argue that EI is trait based will tend towards assuming a certain degree of divergent validity with IQ and convergent validity with personality and will tend to favour self-report tests. It is vital, therefore, that researchers clearly define the terms they employ, to avoid confusion. For instance, Gardner (2006) claims that there have been three distinct meanings applied to the concept of intelligence as follows: (1) Intelligence as a species characteristic (i.e. inherently human); (2) Intelligence as individual difference (i.e. psychometric and therefore measurable); and (3) Intelligence as fit execution of an assignment (i.e. something that can be taught).

With respect to emotional intelligence, certainly many theorists in this area agree that intelligence is an inherent human capacity (although not all, of course, argue that intelligence is solely a human capacity) and most also agree that intelligence can be measured, although there is not full agreement as to the best procedures or techniques to employ. It is the third of Gardner's assertions that is, arguably, the most contentious and that reflects the trait versus ability debate. There are persuasive arguments that have been proposed on both sides of this debate but it would appear that, as with IQ, there are, in fact, both genetic and social determinants of EI. Focusing on the relative impact of biological and social factors in determining individual functioning in this domain, Gardner claims that:

'Once we recognize that intelligence evolves through a dynamic of individuals' competencies and society's values and institutions, we are more likely to devise policies and support initiatives that effectively engage people's minds.' (p. 211).

Based on the argument that trait and ability emotional intelligence are in fact separate, yet related constructs, there has been some attempt in recent years to create unified theories of EI that encompass aspects of both. For example, Daus and Ashkanasy (2005) have argued that ability EI is a distinct, true form of intelligence and trait EI constitutes the capability to apply emotional abilities in everyday contexts i.e. they propose that ability EI refers to the raw talent one may possess with respect to emotional and social reasoning and trait EI to the extent to which that raw talent is 
utilised. In this regard, trait and ability EI would be considered as complimentary rather than contradictory as per Emmerling's claim, above.

As well as facing some difficulty with respect to the construct validity of EI, researchers have also identified some issues with respect to the measurement and interpretation of EI scores. One such issue is that, at present, the stability of EI has not been measured in any meaningful way (Matthews, Zeidner and Roberts 2008). For example, do individuals display high EI in certain situations only and if so, are there specific social or other factors (e.g. age) which enable or inhibit EI? This issue is particularly pertinent to research that is designed to be predictive, for example, in educational contexts, or where EI profiles may be used as an aid to personnel selection or to assess leadership potential. However, thus far, research has not addressed this issue in any meaningful way.

Vachon and Bagby (2008 in Matthews, Zeidner and Roberts 2008) also argue that, in certain situations or certain combinations, higher EI scores, rather than being of benefit, may in fact convey significant physical and mental health risks. For example, they found that participants higher in emotional perception also had higher levels of depression and hopelessness and they argue, accordingly, that certain combinations of high and low EI scores may be particularly detrimental to positive mental health. One example they provide relates to individuals who may possess higher levels of self-awareness, but lower levels of emotional problem solving (i.e. who have the capacity to recognise negative emotions but not to deal with them). Clearly, caution is needed when interpreting EI test scores in order not to cause harm for potential test takers and much further research is required in this area.

There is also some evidence to suggest that tests of EI may be susceptible to faking. Day and Carroll (2008) asked participants to complete tests of EI under two conditions. In the first 'applicant' condition, participants were given a job description and told to complete two separate measures of EI, one interactionist and one ability-based test, as if they were applying for that job. In the second 'non-applicant' condition, participants were asked to complete the measures as honestly as they could. Results revealed that participants would have been more likely to be selected for the job in question based on their 'applicant condition' scores for the interactionist test, but that no differences were found between the 'applicant' and 'nonapplicant' conditions for the ability-based test. Arguably, as this research was conducted under contrived conditions, it may not be entirely reliable. 
However, this issue certainly merits further attention and this research suggests that, at least to some extent, it is possible to fake scores for some tests of EI. This is perhaps not very surprising given that tests of EI involve assessing intra-personal aspects of the human psyche. As the intrapersonal realm is naturally specific to the individual, it is difficult to measure objectively and it is also difficult to verify the responses of test takers (Petrides, Furnham and Mavrovelli 2007). Due to social desirability, the faking of test responses may be particularly likely when feedback is to be given to participants pertaining to their EI profiles or where EI coaching is taking place, as test takers may want to appear to be as emotionally intelligent as possible. This issue may be particularly relevant to educational settings, as students may be fearful of disclosing perceived emotional weaknesses. Therefore careful consideration needs to be given to the manner in which EI coaching takes place in educational environments. This issue is discussed in further detail in chapter four.

Although much research has been conducted with respect to social desirability and personality, very little research, to date, has addressed the issue of social desirability vis-à-vis EI. Kluemper (2008) found that social desirability and core self-evaluations accounted for $62 \%$ of the variance in EI scores for a sample of American employees $(n=180)$. However, once these variables were controlled for, trait EI was still discriminately predictive of life satisfaction, coping and stress. Downey, Godfrey, Hansen and Stough (2006) also found that, for a sample of Australian employees $(n=79)$, social desirability and emotional intelligence were only weakly related. Therefore, preliminary research evidence seems to suggest that, although social desirability does impact test takers' response patterns, that tests of EI still maintain predictive validity once this factor is controlled for. However, much more research is required in this area.

With respect to the manner in which EI profiles are typically generated, as stated previously, those who subscribe to the concept of EI as abilitybased, tend to favour the use of performance-based tests when measuring aspects of EI, whilst those who subscribe to the concept of EI as a trait, tend to favour the use of self-report measures. However, this also raises a number of important questions. For example, if performance-based measures are employed, the assumption is made that there are objective 'correct' responses that exist for test items and, if this is the case, how might agreement be reached as to what exactly those correct responses are? In this regard, Matthews, Emo, Roberts and Zeidner (2013), cited from Murphy K. (2013) claim that, 
The gold standard for conventional intelligence tests is that responses to questions can be scored as 'right' or 'wrong' according to some explicit rationale. In the emotional domain, though, determining the 'correct' answers to questions concerning how best to resolve an argument, comfort a friend, or deal with strong personal emotions is by no means straightforward, especially when there may be many viable solutions' ( $\mathrm{p}$. 5).

However, Matthews, et al. (2008) had earlier stated that '...the scope and importance of the validity coefficients for EI remains open for debate, but the proponents of EI have made progress in demonstrating that the scales have sufficient criterion validity to be taken seriously.' (p. 7). Clearly, much further research is required to resolve this issue. However, although there is still some debate as to the extent to which EI is trait or ability based and how best EI should be measured, sufficient research evidence has been amassed to support the claim that EI is a distinct and measureable form of intelligence. As continued progress has been made with respect to assessing the construct validity of EI, a growing body of research has focused on developing, testing and applying theories of emotional intelligence in academic, organisational and other arenas.

\section{Tests of EI}

A cursory knowledge of the most widely employed tests of emotional intelligence is of benefit to educators or researchers who are interested in developing students' social and emotional competencies. As outlined in the preceding section, theorists are not in agreement as to whether emotional intelligence is trait based, ability based, or a combination of both. Tests that have been developed to measure EI are similarly divided, with different tests being predicated on the assumption that EI is ability or trait based. At present, there are a number of competing tests of emotional intelligence, not all of which have received the same level of interest or which have been empirically evaluated and this is another factor that must be considered when research is being conducted in educational contexts.

The number of tests of emotional intelligence has grown in recent years, which highlights the increasing attention being given to this field as well as the increase in the amount of research being conducted with respect to emotional intelligence. For example, the Encyclopaedia of Applied Psychology (Speilberger 2004) stated that there were three principal tests of emotional intelligence which were currently in use at that time; the MSCEIT, the ESCI-2 and the EQ-i. More recently, the Consortium for 
Research in Emotional Intelligence in Organizations (2015), has compiled a list of tests in current usage for which there has been a substantial body of research (a minimum of five journal articles or book chapters that provide empirical data related to the test in question). They name nine such separate tests of EI. Of these, three are designed specifically for use in business settings (the Work Group Emotional Intelligence Profile, the Genos Emotional Intelligence Inventory and the Group Emotional Competence Inventory) and will therefore not be discussed further as they are not ordinarily employed in educational settings. Each of the six other tests that are listed by the consortium are outlined below. However, it is also important to note that, although there are competing tests and competing concepts of emotional intelligence, they tend to be 'complimentary rather than contradictory' (Emmerling 2007, 73).

\section{Mayer-Salovey-Caruso Emotional Intelligence test (MSCEIT)}

This particular test was devised in the early 1990s and takes an 'ability approach' which considers EI as a traditional form of intelligence. As the authors of this test believe EI to be ability based, the test is a performance measure. The test consists of eight sub-tests divided into four branches; Identifying emotions, emotional facilitation, understanding emotions and managing emotions. Respondents must, for example, recognise emotions on faces in pictures and identify the feelings that are evoked by specific provocative pictures. Respondents must also identify feelings which may interfere with performance in a number of modalities, analyse blended emotions and appreciate techniques that may be useful for managing either one's own emotions or the emotions of others. The 141-item test is designed so that there are more or less correct answers to each test item and, unusually, there are two separate scoring tables that are available for this test. Respondents' answers may be judged either against answers given by an original sample of emotion experts $(n=21)$ from the 'International Society for Research on Emotions', or answers that are derived by 'consensus' in which case they are compared to answers given by an original sample $(n=5000)$ designed to measure how the 'average' person responds. This particular test has strong internal consistency and test-retest reliability (Brackett and Mayer 2003). However, concerns have been raised with regard to the comparison of scores against those of the original samples and that it measures the 'norm' and biases against those with significantly high levels of EI. In effect, critics argue that this bias occurs as only responses that were endorsed by a majority of the original 